\title{
Fractional Analysis of Ascorbic Acid and Dopamine by Copper Nanoparticles Electrodeposited onto Gold Electrode
}

\author{
MOHAMED I. AWAD ${ }^{1,2^{*}}$, MOHAMMED A. KASSEM ${ }^{1,3 *}$, AHMED M. HAMEED ${ }^{1}$, \\ B.A.AL JAHDALI ${ }^{1}$ and OMAR A. HAZAZI ${ }^{1}$
}

${ }^{1}$ Chemistry Department, Faculty of Applied Sciences, Umm Al-Qura University,
Makkah Al-Mukarramah, Saudi Arabia Kingdom.
${ }^{2}$ Chemistry Department, Faculty of Science, Cairo University, Cairo, Egypt.
${ }^{3}$ Chemistry Department, Faculty of Science, Benha University, Benha13518, Egypt.
${ }^{*}$ Corresponding author E-mail: mawad70@yahoo.com, maa_kassem @ hotmail.com

http://dx.doi.org/10.13005/ojc/330421

(Received: May 18, 2017; Accepted: July 10, 2017)

\begin{abstract}
The electrochemical behavior of ascorbic acid (AA) and dopamine (DA) at copper nanoparticles modified polycrystalline gold electrode (nano-Cu/Au) has been studied using cyclic voltammetry. The modified nano-Cu/Au electrodes have been assembled using cyclic voltammetric techniques. Nano-Cu/Au have been successfully differentiated the electrochemical response of the two species, in contrary to the bare polycrystalline gold electrode at which the electrochemical response of the two species were overlapped, and only one anodic peak for the two species is obtained. At the optimized conditions, both species have been simultaneously determined using square wave voltammetry. Acceptable rectilinear ranges for both species have been obtained with correlation coefficients of 0.998 and 0.995 for DA and AA, respectively. The limits of detection and quantification (LOD and LOQ) were calculated and found to be 1.816 and 6.05 for DA and 34.57 and 115.25 for AA. The relative standard deviation (RSD) for both DA and AA were deliberated to be 2.04 and $0.376 \%$, respectively.
\end{abstract}

Keywords: Dopamine, Ascorbic acid, Voltammetry, Modified Gold Electrode, Electroanalysis.

\section{INTRODUCTION}

Metal nanoparticles with their unique electrocatalytic properties, compared with their metal counterparts, have been applied in a wide spectrum of applications ${ }^{1-9}$. They have size dependent unique optical, electrical and chemical properties and are very hopeful for everyday applications in various research areas, like molecular catalysts, multifunctional reagent and biosensors. In many cases, the sluggish electrochemical behavior of several compounds was enhanced via the tailored 
nanoparticles modified electrodes, and also for the solution of the fouling of some bulk materials ${ }^{5,9}$. For example, the electrochemical behavior of dopamine (DA) at polycrystalline gold electrode is unstable, and after few cycles at poly-Au electrodes, the electrode is fouled and the electro-oxidation of the dopamine is largely retarded. Fouling has been reported to be due to the adsorption of the oxidation product. In addition dopamine analysis cannot be realized at the bare poly-gold electrode in the presence of the coexisting ascorbic acid as their electrochemical response can't be differentiated ${ }^{10}$.

Separation of the oxidation potentials of the two peaks have been realized at gold modified electrodes, whether modified by organic or inorganic modifier. Several papers have been published regarding the analysis of DA and AA in their coexistence at thiol self-assembled monolayer onto gold electrodes ${ }^{11,12}$. The separation of the two peaks relies on the differential interaction of the two species, i.e., DA and AA with the modified electrodes. The electrochemical behavior of one species was enhanced while the other one was either retarded or unaffected $^{11,12}$. However, those electrodes suffer the desorption of the modifier, and consequently loose its electrocatalytic activity, especially when subjected to higher anodic potentials. Gold electrodes modified with inorganic species have presented more stable behavior with high electrocatalytic activity, especially when modified in the nanoscale size ${ }^{13-18}$.

Several methods for the determination of DA are available in literature. It includes, but not limited to spectrophotometry, capillary electrophoresis and high performance liquid chromatography ${ }^{19-23}$. However, these methods suffer several drawbacks that can be solved by electrochemical methods known by simple setting. In the present work DA and AA analysis in their coexistence at copper nanoparticles modified

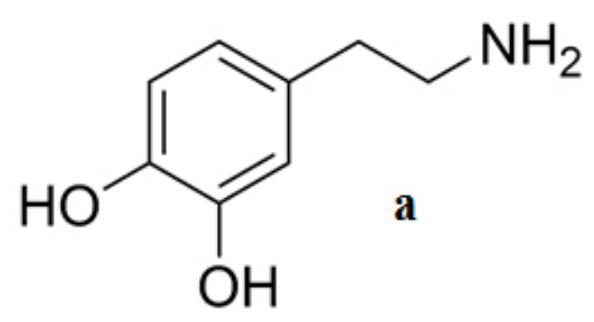

gold electrodes will be studied. This combination is selected as gold electrode is well known to reveal the two peaks for the oxidation of the two species at an acceptable potential range, and copper is expected to solve the problem of fouling via giving an alternative site for the adsorption of the oxidation products. The experimental conditions for a selective and sensitive analysis are optimized.

\section{EXPERIMENTAL}

\section{Chemicals}

Analytical grade chemicals were used in all experiments which prepared using bidistilled water. Standard solutions of ascorbic acid and dopamine, obtained from Sigma-Aldrich, with molecular formulas shown in Fig. 1 were freshly prepared by dissolving claculated amounts of them in bidistilled water. The phosphate buffer solutions of different $\mathrm{pHs}$ were prepared according to Gorimi ${ }^{24}$ and then adjusted using a $\mathrm{pH}$-meter.

\section{Electrodes}

The polycrystalline gold electrode (poly$\mathrm{Au})(0.16 \mathrm{~cm}$ in diameter) has been prepared as previously reported $[2,4]$. Shortly, it is polished to a mirror-like with alumina powder and emery paper (down to $0.06 \mathrm{~mm}$ ), and then rinsed using ultrasonic waves in bidistilled water. Next, it is electrochemically pretreated in nitrogen-saturated $0.05 \mathrm{M}$ sulfuric acid solution by repeating the potential scan in the potential ranges $(-0.2-1.5 \mathrm{~V}) \mathrm{vs}$. $\mathrm{Ag} / \mathrm{AgCl}(\mathrm{KCl}$ sat.) until the cyclic voltammogram finger print of a clean gold electrode was attained.

\section{Elaboration of the modified electrode Nano-Cu/ Au \\ Cupper nanoparticles (nano-Cu) modified} $\mathrm{Au}$ electrode (nano-Cu/Au) was prepared by the deposition of nano-Cu electrochemically onto the

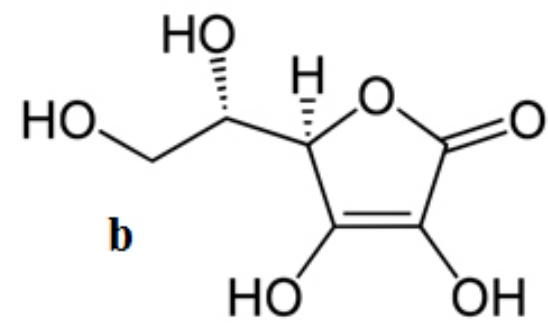

Fig. 1: The structure of (a) Dopamine and (b) Ascorbic acid 
pretreated polycrystalline gold electrode using $0.1 \mathrm{M}$ phosphate buffer solution of $\mathrm{pH} 7$ containing $1.0 \mathrm{mM}$ $\mathrm{CuSO}_{4}$ by conducting one or more potential cycle at a scan rate of $50 \mathrm{mV} \mathrm{s}^{-1}$ between 0.7 and $-0.2 \mathrm{~V}$ vs. $\mathrm{Ag} / \mathrm{AgCl}$ ( $\mathrm{KCl}$ sat.).

\section{Measurement}

The electrochemical measurements were carried out using a PGSTAT30 (Netherlands) galvanostat/potentiostat controlled by software of (FRA) and (GPES). An Ag/AgCl ( $\mathrm{KCl}$ sat.) electrode was used as the reference electrode. The counter electrode (a platinum spiral wire) and the working electrode were separated by a porous glass. A conventional three-electrode glass cell of about $40 \mathrm{ml}$ capacity was used for all measurements. All electrochemical measurements were conducted under nitrogen saturated solutions.

\section{RESULTS AND DISCUSSION}

\section{Voltammetric characterization}

The morphological and voltammetric behavior of the copper nanoparticles modified polycrystalline Au electrode (nano-Cu/Au) has been previously reported ${ }^{4}$. The voltammetric behavior of nano-Cu/Au has been marked by conducting cyclic voltammetry in $\mathrm{N}_{2}$-saturated $0.5 \mathrm{M}$ sulfuric acid, and the deposition of nano copper has been revealed from the decrease in the peak correspond to the formation of gold oxides monolayer and its couple corresponding to the reduction peak ${ }^{2}$. The

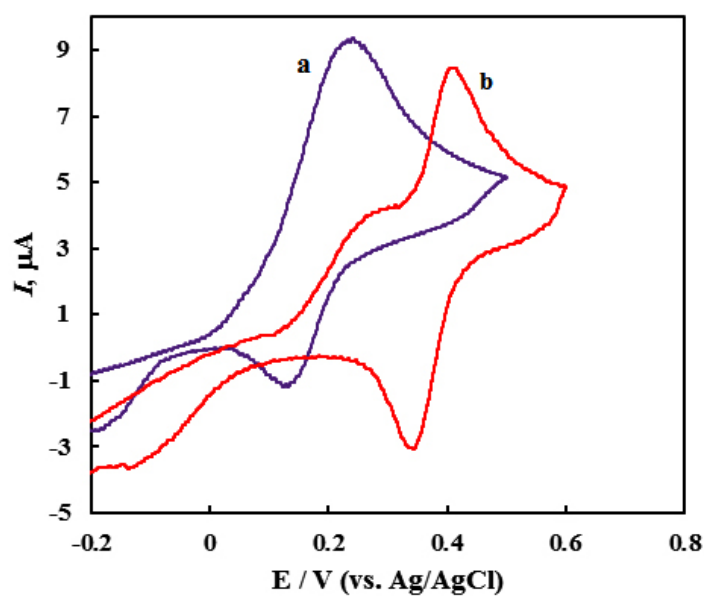

Fig. 2: CVs obtained at (a) Au and (b) nano-Cu/ Au electrodes in in $\mathrm{N}_{2}$-saturated $0.1 \mathrm{M}$ phosphate buffer pH 7 containing $500 \mu \mathrm{M}$ DA and $1 \mathrm{mM}$ AA surface areas of both electrodes were estimated from the charge consumed in the reduction peak of the gold oxide monolayer, not from the reduction peak, to avoid the overestimation in the case of using oxidation peak as a result of the overlapping of this peak with the oxygen evolution. The surface coverage, $\theta$, of the deposited Cu nanoparticles, was also calculated from the following equation ${ }^{25}$;

$$
\theta=1-Q_{1} / Q_{2}
$$

where $Q_{1}$ is the amount of charge consumed in the gold oxide reduction peaks obtained at the nano-Cu/Au electrode and and $Q_{2}$ the amount of charge consumed in the gold oxide reduction peaks obtained at bare Au electrode. It has been found that, surface coverage, $\theta=0.22$ pointing to the incomplete covering of the underlying gold substrate by nano$\mathrm{Cu}$, consistently with the reported AFM image ${ }^{4}$.

Fig. 2 represents CVs obtained at bare polycrystalline gold (a), and nano-Cu/Au (b) electrodes in $\mathrm{N}_{2}$-saturated phosphate buffer solution of $\mathrm{pH} 7.0$ containing $1.0 \mathrm{mM} \mathrm{AA}+0.5 \mathrm{mM} \mathrm{DA}$. At bare polycrystalline $\mathrm{Au}$ electrode (curve a) one anodic peak corresponding to the electrochemical responses of DA and $A A$ over a wide potential range (ca. 0-0.5 V). This anodic peak is coupled by one cathodic peak centered at $0.16 \mathrm{~V}$, probably corresponds to the dopamine reduction as ascorbic

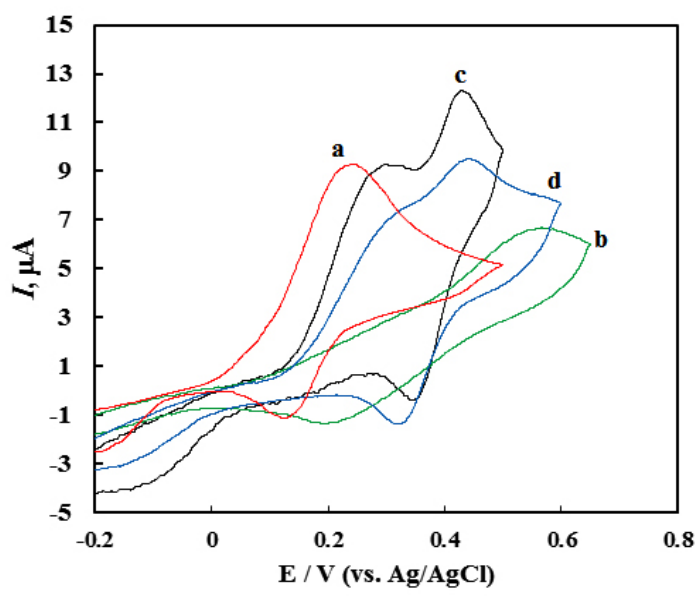

Fig. 3: CVs obtained at (a) Au and (b, c, d) nano-Cu/Au electrodes in in $\mathrm{N}_{2}$-saturated $0.1 \mathrm{M}$ phosphate buffer pH 7 containing $500 \mu \mathrm{M}$ DA and $750 \mu \mathrm{M}$ AA, (b) one, (c) two and (d) five potential cycles 
clearly shown, the loading level of $\mathrm{Cu}$ is inherently controlling; while nano-Cu/Au electrode prepared by two potential cycles nicely separate enough the two oxidation peaks of DA and $A A$ for furher analysis of the two species in their coexistence, the one prepared by one potential cycle didnot separate the two oxidation peaks, and rather one broad peak is obtained. The one prepared by 5 potential cycles (curve d) though it could separate the two peaks, but

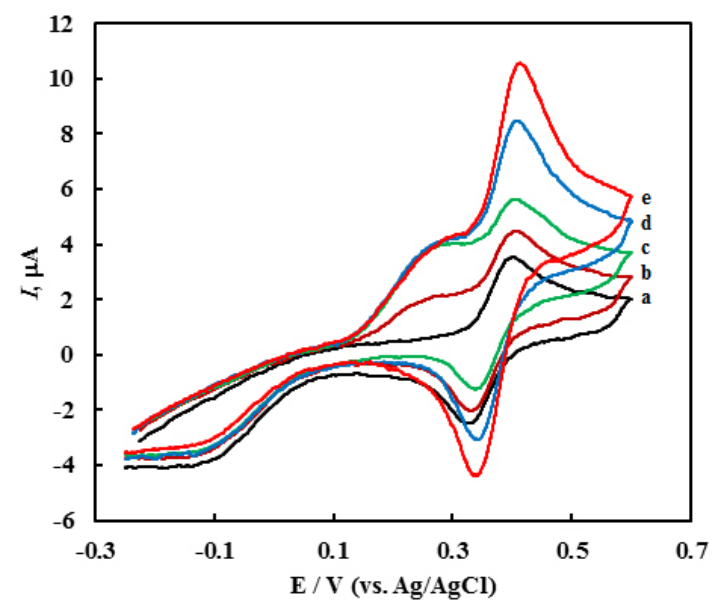

Fig. 5: CVs obtained at modified nano-Cu/Au electrode in in $\mathrm{N}_{2}$-saturated $0.1 \mathrm{M}$ phosphate buffer pH 4.5 containing $250 \mu \mathrm{M}$ DA (a), $250 \mu \mathrm{M}$ DA - $250 \mu \mathrm{M}$ AA (b), $250 \mu \mathrm{M}$ DA - $500 \mu \mathrm{M}$ AA (c), $500 \mu \mathrm{M}$ DA - $500 \mu \mathrm{M}$ AA (d) and $500 \mu \mathrm{M}$ DA $-750 \mu \mathrm{M}$ AA (d)

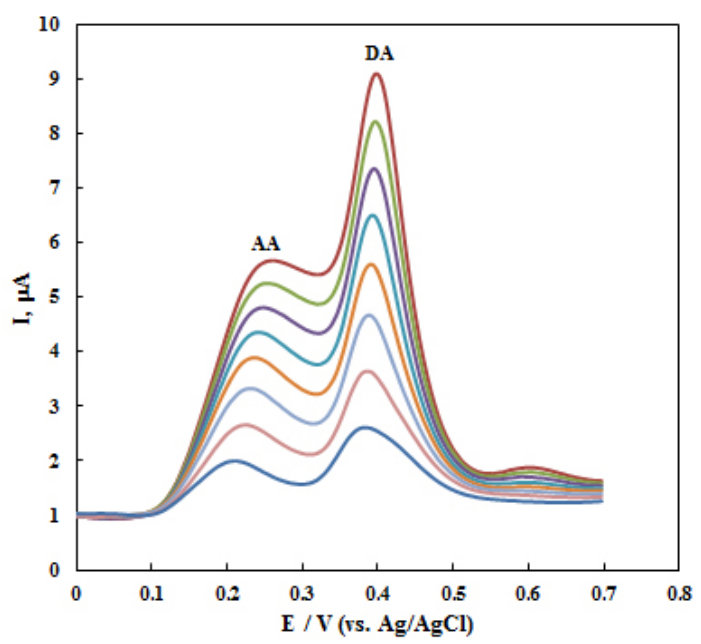

Fig. 6. Square wave voltammograms obtained at modified $\mathrm{Cu} / \mathrm{Au}$ electrode for simultaneous determination of DA and AA in phosphate buffer (pH 4.5) the separation is not as much as the one obtained in the case of the modified electrode prepared by 2 potential cycles. Moreover, the current is this case is smaller comparatively. Hence, the loading prepared by two potential cycles will be used hereafter as the optimum one.

Next the pH effect is optimized, the oxidation of DA (Eq. 2) and AA (Eq. 3) involves protons and thus it is expected to be $\mathrm{pH}$ dependent. CVs of DA and $\mathrm{AA}$ obtained at nano-Cu/Au electrode at a scan rate of $100 \mathrm{mV} \mathrm{s}^{-1}$ in phosphate buffer solutions of dissimilar pHs are shown in Fig. 4. As clearly shown, as expected, the peak potentials of the two species shifted negatively upon increasing the $\mathrm{pH}$. In addition the peak current slightly increased. The large peak separation is obtained at $\mathrm{pH} 4.5$ (curve a). Larger than this $\mathrm{pH}$ the two peaks for the DA and AA oxidation overlap. Thus the $\mathrm{pH}$ of 4.5 will be used hereafter as the optimum one along with the loading obtained by two potential cycles. Peaks potential extracted from this figure are plotted against $\mathrm{pH}$, and a linear plot with a slope equals $60 \mathrm{mV} / \mathrm{pH}$ was obtained (data are not shown). The linear dependence of peak potential of DA on $\mathrm{pH}$ indicates the presence of a proton-transfer reaction preceding the electrode process. The slope is nearby to that for a Nernstian reaction, revealing that the uptake of 2 electrons is accompanied by an equal number of protons (Eqs. 2 and 3). Similar results are obtained for the dependence of the oxidation of AA on electrolyte $\mathrm{pH}$.

The selectivity of the electroanalysis for DA and AA presented here, as a main criteria for the analysis of the two species in their coexistence was investigated, by measuring the CVs at the nano-Cu/Au working electrode for $0.25 \mathrm{mM}$ DA in the presence of different amounts of ascorbic acid (Fig 5). DA oxidation peak current keeps constant when the concentration of DA was kept constant while changing the concentration of AA. Also same behavior with keeping the concentration of $A A$ and changing the concentration of DA; the former oxidation peak keeps constant while the latter changes. At nano-Cu/Au electrode, AA oxidation peak is negatively shifted compared with its oxidation at bare electrode. This enables the selective analysis of DA in the presence of AA. 

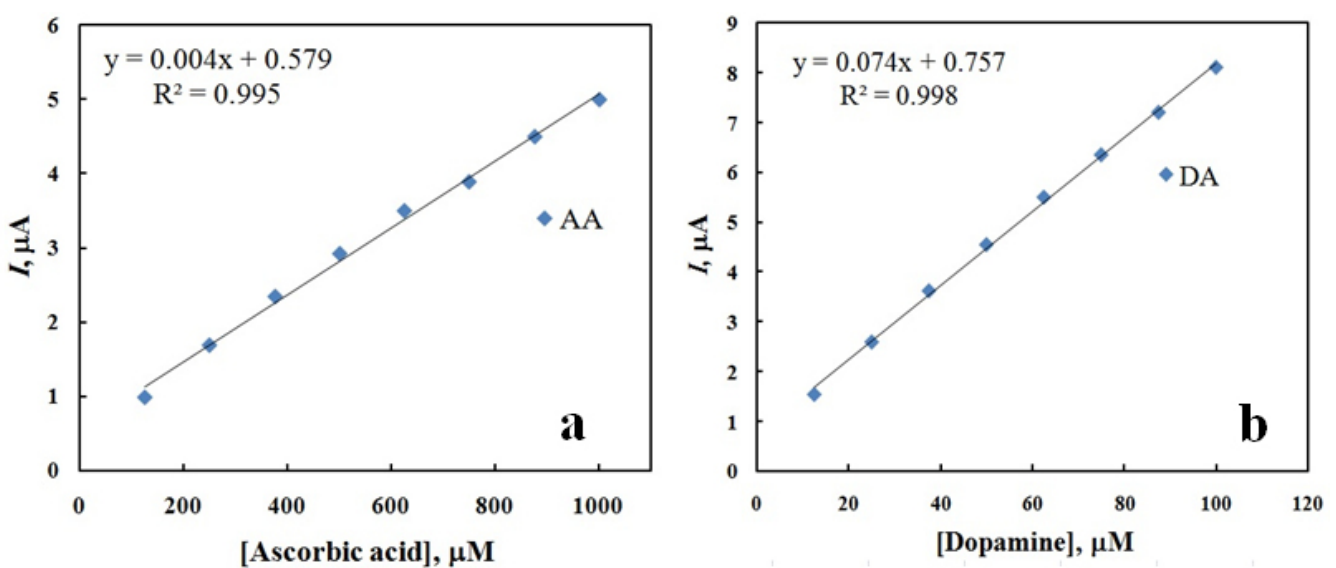

Fig. 7: Calibration curves for both (a) ascorbic acid and (b) dopamine extracted from square wave voltammetry (SWs) using phosphate buffer solution of $\mathrm{pH} 4.5$

\section{Square wave voltammetry}

The two well-separated square wave voltammograms peaks for AA and DA oxidation are utilized for their selective determnation. Square wave voltammetry (SWV) offers excellent discrimination against double-layer charging current and accordingly has a high sensitivity ${ }^{26,27}$ compared with cyclic voltammetry. First the interrelated instrumental parameters, i.e., step potential, square wave amplitude and frequency, were optimized before recording the square wave voltammograms for the oxidation of DA and AA. Finally the following parameters were used: step potential, $4 \mathrm{mV}$, square wave amplitude, $25 \mathrm{mV}$, and frequency $25 \mathrm{~Hz}$ (and thus the scan rate is $100 \mathrm{mV} \mathrm{s}^{-1}$ ).

The SWV of AA and DA in their coexistence were recorded at nano-Cu/Au electrode at the optimum conditions of $\mathrm{pH}$ and instrumental parameters and results are shown in Fig. 6 in which well-separtated SWVspeaks are obtained for the oxidation of successive addition of $A A$ and DA. The oxidation peaks of DA and AA are noticeably detected at about 0.38 and $0.22 \mathrm{~V}$, respectively. Based on the SWVs obtained, the linear calibration curves for DA and AA were obtained in $\mathrm{N}_{2}$-saturated phosphate buffer solution of $\mathrm{pH} 4.5$ over a wide concentration range and shown as Fig. 7. The relative standard deviation (RSD) was calculated and found to be 2.04 and $0.376 \%$ for DA and AA, respectively, representing a perfect precision of the studied routine.
The limit (LOD) and quantification (LOQ) of detection are estimated based on Eqs. 4 and 5, respectively ${ }^{28}$ :

$\mathrm{LOD}=3 \mathrm{~s} / \mathrm{m}$

$\mathrm{LOQ}=10 \mathrm{~s} / \mathrm{m}$

where $s$ is the standard deviation of the response (s) and $m$ is the slope of the calibration curve. The LOD and LOQ for the present electroanalysis of DA were calculated as 1.816 and 6.05 micromolar, respectively and for AA as 34.575 and 115.25 micromolar, respectively. Analytical parameters for simultaneous determination of DA and $A A$ at the nano-Cu/Au electrode are summarized in Table 1. Relative standard deviation is of smaller value pointing to the high reproducibility of the present method. In addition statistical data obtained using F-test shows the high significance of the results obtained by the present method ${ }^{29}$.

\section{CONCLUSIONS}

Dopamine (DA) and ascorbic acid (AA) voltammetric behaviors have been studied at nanoCu modified polycrystalline (poly-Au) electrode. DA and AA has been selectively and simultaneously analyzed at nano-Cu modified polycrystalline gold electrode (nano-Cu/Au) in phosphate buffer solution $(\mathrm{pH}$ 4.5). The loading level of $\mathrm{Cu}$ as 
well as the oxidation of the deposited nano-Cu have played a prominent role in the simultaneous determination of the two species. The calibration curves for both species have been obtained over an acceptable concentration range with a high correlation coefficient

\section{ACKNOWLEGMENT}

The authors would like to thank Institute of Scientific Research and Revival of Islamic Heritage at Umm Al-Qura University (Project ID 43405072) for the financial support.

\section{REFERENCES}

1. Etesami, M; Norita, M; Int. J. Electrochem. Sci., 2011, 6, 4676-4689.

2. Awad, M.I; El-Deab, M.S; Ohsaka, T. J. Electrochem. Soc., 2007, 154, 810-816

3. Danial, A.S; Awad, M.I; Al-Odail, F.A; Saleh, M.M. J Mol Liqu. 2017, 227, 919-925.

4. Kassem, M.A; Hazazi, O.A; Ohsaka, T; Awad , M.I. Electroanalysis, 2016, 27, 539-545.

5. Awad, M.I; Saleh, M.M; Ohsaka T. J Solid State Electrochem. 2015, 19, 1331-1340.

6. El-Refaei, S.M; Saleh, M.M; Awad, M.I. J Solid State Electrochem. 2014, 18, 5-12

7. El-Refaei, S.M; Saleh, M.M; Awad, M.I. J. Power Sources 2013, 223, 125-128.

8. El-Refaei, S.M; Awad, M.I; El-Anadouli, B.E; Saleh, M.M. Electrochimica Acta 2013, 92, 460-467.

9. Awad, M.I; Ohsaka, T. J. Power Sources 2013, 226, 306-312.

10. Capella, P; Ghasemzadeh, B; Mitchell, K; Adams, R.N. Electroanalysis, 1990, 2, 175182.

11. Raj, C.R; Oshaka, T. Electrochem. Commun., 2001, 11, 633-638.

12. Raj, C. R; Ohsaka, T. Bioelectrochem., 2001, 53, 251-256.

13. Liu, L; Du, J; Li, S; Yuan, B; Han, H; Jing, M; Xia, N. Biosens. Bioelectron. 2013, 41, 730735.

14. Kaya, M; Volkan, M. Anal. Chem. 2012, 84, 7729-7735.

15. Weng, S.H; Zheng, Y.J; Zhao, C.F; Zhou, J.Z; Lin, L.Q; Zheng, Z.F; Lin, X.H. Microchim. Acta. 2013, 180, 371-378.
16. Li, Y; Huang, F; Chen, J; Mo, T; Li, S; Wang, F; Feng, S; Li, Y. Int. J. Electrochem. Sci. 2013, 8, 6332-6342.

17. Yu, H.Y; Xu, M.Q; Yu, S.H; Zhao, G.C. Int. J. Electrochem. Sci. 2013, 8, 8050-8057.

18. Wang, H.B; Zhang, H.D; Chen, Y; Huang, K.J; Liu, Sens. Y.M. Actuators B: Chem. 2015, 220, 146-154.

19. Zhao, Y.S; Zhao, S.L; Huang, J.M; Ye, F.G. Talanta 2011, 85, 2650-2654.

20. Jacobus, F.S; Raluca, S.S. Talanta 2012, 102, 34-43.

21. Attilio, N; Emanuela, G; Giovanni, S; Antonio, T. Anal. Chim. Acta 2014, 810, 17-24.

22. Gottas, A; Ripel, A; Boix, F; Vindenes, V; Mørland; J; Øiestad, E.L. J. Pharmacol. Toxicol. Meth. 2015, 74, 75-79.

23. Ferry, B; Gifu, E.P; Sandu, I; Denoroy, L; Parrot, S. J. Chromatogr. 2014, 951, 52-57.

24. Gomori, G. "Methods in Enzymology”, 1955, 1,141 .

25. Trasatti, S; Petrii, O.A. Pure Appl. Chem. 1991, 63, 711-734.

26. O’Dea, J.J; Osteryoung, J; Osteryoung, R.A. Anal. Chem., 1981, 53, 695-701.

27. Validation of Analytical Procedures, Methodology, International Conference on Harmonization (ICH), 1994,6.

28. Murray, R.W. In Electroanalytical Chemistry, ediyed by A J Bard \& Stratmann, (Marcel Dekker, New York) 1984, 13,191.

29. Qu, W; Wu, K; Hu, S; J. Pharm. Biomed. Anal. 2004, 36, 631-635. 